\title{
Co-evolutionary dynamics in social networks: a case study of Twitter
}

\author{
Demetris Antoniades ${ }^{1 *}$ and Constantine Dovrolis ${ }^{2}$
}

\author{
${ }^{*}$ Correspondence: \\ danton@cs.ucy.ac.cy \\ ${ }^{1}$ Department of Computer Science, \\ University of Cyprus, Nicosia, Cyprus \\ Full list of author information is \\ available at the end of the article
}

\begin{abstract}
Complex networks often exhibit co-evolutionary dynamics, meaning that the network topology and the state of nodes or links are coupled, affecting each other in overlapping time scales. We focus on the co-evolutionary dynamics of online social networks, and on Twitter in particular. Monitoring the activity of thousands of Twitter users in real-time, and tracking their followers and tweets/retweets, we propose a method to infer new retweet-driven follower relations. The formation of such relations is much more likely than the exogenous creation of new followers in the absence of any retweets. We identify the most significant factors (reciprocity and the number of retweets that a potential new follower receives) and propose a simple probabilistic model of this effect. We also discuss the implications of such co-evolutionary dynamics on the topology and function of a social network. Finally, we briefly consider a second instance of co-evolutionary dynamics on Twitter, namely the possibility that a user removes a follower link after receiving a tweet or retweet from the corresponding followee.
\end{abstract}

Keywords: Online social networks; Complex networks; Co-evolution

\section{Introduction}

Online social networks (OSNs), such as Twitter and Facebook, have changed how individuals interact with society, how information flows between actors, and how people influence each other. These are all complex dynamic processes that are now widely studied empirically and in a large scale, thanks to the availability of data from OSNs. Most OSN studies focus on one of the following two aspects of network dynamics. Dynamics on networks refer to changes in the state of network nodes or links considering a static topology $[1,2]$. Dynamics of networks, on the other hand, refer to changes in the topology of a network, without explicitly modeling its underlying causes [3]. As noted by Gross and Blasius in [4], however, real OSNs typically exhibit both types of dynamics, forming an adaptive, or co-evolutionary, system in which the network topology and the state of nodes/links affect each other through a (rather poorly understood) feedback loop.

Dynamic processes in OSNs, such as information diffusion or influence, are obviously affected by the underlying network topology, but they also have the power to affect that topology. For instance, users may decide to add or drop a "friendship" or "follower" relation depending on what the potential "friend" or "followee" has recently said or done in the context of that OSN. Previous empirical or modeling OSN studies often choose to

(c) 2015 Antoniades and Dovrolis. This is an Open Access article distributed under the terms of the Creative Commons Attribution License (http://creativecommons.org/licenses/by/4.0), which permits unrestricted use, distribution, and reproduction in any medium, provided the original work is properly cited. 
ignore such co-evolutionary dynamics, mostly for simplicity, assuming a static network topology, or assuming that the topology and node/link states are decoupled and evolve in separate time scales [5].

In this paper, we focus on co-evolutionary dynamics in the context of Twitter. Twitter users create follower-followee relations with each other. A directed link from a user $R$ to a user $S$, denoted by $R \rightarrow S$, means that $R$ is a follower of $S$, receiving $S$ 's tweets; $S$ is referred to as a followee of $R$. $R$ can choose to propagate a tweet of $S$ to her own followers, denoted by $F(R)$, creating a retweet. When a follower $L \in F(R)$ receives a retweet of $S$ through $R, L$ can choose to add $S$ to her followers. We call this sequence a Tweet-Retweet-Follow (TRF) event, and refer to its three main actors as Speaker S, Repeater $R$, and Listener $L$. TRF events represent a clear case of co-evolutionary dynamics: information propagation (tweet-retweet) causes a topology change (new follower).

Figure 1 shows this sequence of events for the simplest TRF case in which $R \rightarrow S$ and $L \rightarrow R$. In general, the Repeater $R$ may not be a follower of $S$ but she may receive $S$ 's tweet through a cascade of retweets. Additionally, the Listener $L$ may receive multiple retweets of $S$ from the same or from different Repeaters. The contributions of this study are as follows:

1. We propose a measurement approach to detect TRF events, based on near real-time monitoring of a Speaker's activity and followers.

2. We show that the formation of new follower relations through TRF events is orders of magnitude more likely than the exogenous arrival of new followers in the absence of any retweets.

3. We identify the most significant factors for the likelihood of a TRF event: reciprocity (i.e., is Speaker $S$ already following Listener $L$ ?), number of received retweets (i.e., how many retweets of $S$ were received at $L$ during a given time interval $\Delta$ ), and of course the interval $\Delta$ itself.

4. We propose a simple but accurate two-parameter model to capture the probability of TRF events.

5. We discuss the implications of TRF events in the structure and function of social networks.

6. We briefly consider a second instance of co-evolutionary dynamics on Twitter, namely the possibility that a user removes a follower link after receiving a tweet or retweet from the corresponding followee.

This paper is an extended version of work published in [6]. We extend our previous work by examining a second instance of co-evolutionary dynamics on Twitter, namely the possibility of an unfollow event to occur.

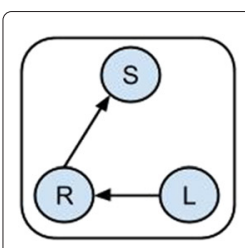

(a) Initial State

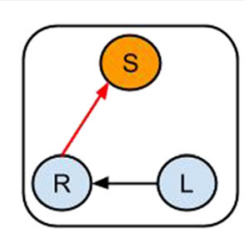

(b) $t_{0}: S$ tweets $M$

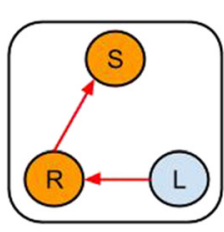

(c) $t_{1}: R$ retweets $M$

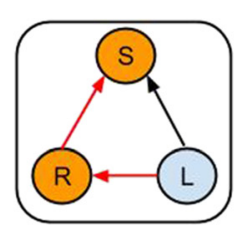

(d) $t_{2}: L$ follows $S$

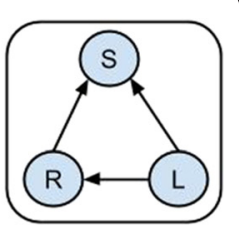

(e) Final state

Fig. 1 a-e Network co-evolution: a Tweet-Retweet-Follow event 


\section{Related work}

Preferential attachment [7] is a common way to think about the formation of new ties in a social network. It is based on the idea that it is more likely for well-connected people to attract new ties. Subsequent research provided a deeper understanding by exploring mechanisms such as user similarity [8, 9] (homophily) and directed closure [10-12]. For instance, Romero and Kleinberg [12] studied the directed closure process in Twitter. This process states that there is an increased likelihood for a node $\mathrm{A}$ to follow a node $\mathrm{C}$ if there already exists a direct path of length two from $\mathrm{C}$ to $\mathrm{A}$. They showed that this process is taking place at a significantly higher rate than what would be expected by chance, but this rate also varies significantly among different users. Here, we identify TRF events as a plausible mechanism for the emergence of directed closure. Further, we examine the factors that affect the probability of closure, offering a plausible explanation for the high variability across users.

Golder and Yardi conducted a user study to identify structural predictors for tie formation in Twitter. Their results show that lack of transitivity has a negative effect in link prediction [10]. Hopcroft et al. examined the question: "when you follow a particular user, how likely will she follow you back?" [8]. They showed that geographic distance and homophily are good predictors of follow-back ("reciprocal") relations. Our work confirms that reciprocity amplifies significantly the likelihood of TRF events.

Muchnik et al. examined the correlation between a user's degree and activity, and found that activity has a causal increasing effect on degree [13]. Our analysis is related, showing that the number of retweets of a user $S$ that user $L$ receives increases the probability that $\mathrm{L}$ will follow $\mathrm{S}$. Leskovec et al. studied network evolution of four social networks and observed that most edges are local, "closing triangles" in particular [11]. Gallos et al. examined the formation and evolution of social networks and analyzed how reciprocity and social balance affect what we refer to as TRF probability [14].

Information diffusion on Twitter has also received significant attention. Several events have shown the major role that Twitter plays in amplifying and spreading information across the globe $[15,16]$. Romero et al. [17] analyzed ways in which socially sensitive topics, including politics, propagate on Twitter and reported that such topics are more likely to spread after multiple exposures than others. Myers et al. [18] examined how information reaches a user in Twitter. By analyzing URL mentions, they discovered that information tends to "jump" across the network (probably because users discover this information from external sources).

The literature on co-evolutionary dynamics has relied mostly on abstract models so far, without sufficient empirical validation. For instance, Kosma and Barrat examined how the topology of an adaptive network of interacting agents and of the agents' opinions can influence each other [19]. When agents rewire their links in a way that depends on the opinions of their neighbors, the result can be either a large number of small clusters, making global consensus difficult, or a highly connected but polarized network. Shaw and Schwartz [20] examined the effects of vaccination in static versus adaptive networks. Interestingly, they show that vaccination is much more effective in adaptive networks, and that two orders of magnitude less vaccine resources are needed in adaptive networks. Volz and Mayers studied epidemics in dynamic contact networks and showed that the rate at which contacts are initiated and terminated affects the disease reproductive ratio [21]. They concluded that static approximations of dynamic networks 
can be inadequate. Rocha et al. simulated epidemics in an empirical spatio-temporal network of sexual contacts [22], showing that dynamic network effects accelerate epidemic outbreaks. Perra et al. studied the effect of time-varying networks in random walks and search processes [23]. The behavior of both processes was found to be "strikingly different" compared to their behavior in static networks.

The most relevant prior work, by Weng et al., analyzed the complete graph and activity of Yahoo! Meme, ${ }^{1}$ to identify the effect of information diffusion on the evolution of the underlying network [24]. They show that information diffusion causes about $24 \%$ of the new links, and that the likelihood of a new link from a user $\mathrm{X}$ to a user $\mathrm{Y}$ increases with the number of Y's posts seen by X. More recently, Myers and Leskovec showed that Twitter users gain or loose bursts of followers soon after their tweet activity event [25]. These bursts increase both the density of connections between a user's followers and the similarity of a user with her followers. Similarly to our work, they show that $21 \%$ of all new follows are formed by users who recently saw a retweet of the target user.

\section{Data collection}

This section explains the data collection process in detail.

To identify TRF events, we need to observe the appearance of a new follower link from an arbitrary Listener $L$ to a monitored Speaker $S$, shortly after $L$ has received a retweet of $S$ through a Repeater $R$. This requires information about both the time of the retweet(s) as well as the time the new follower link has appeared. The Twitter API, though extended in functionality, does not provide information about the creation time of follower relations. Furthermore, existing link creation time inference methods [26] are not applicable in our study because they cannot be used in real time. To retrieve (near) real-time timing, we have implemented a Twitter data retrieval system that periodically checks for new followers and retweets in a given set of Speakers. An overview of our data collection process is shown in Fig. 2. We explain each step of the process in the following paragraphs.

\section{Selection of active Speakers}

We obtain a number of active Twitter users as potential Speakers through a stratified sampling method. It has been reported that about $25 \%$ of Twitter users have never posted

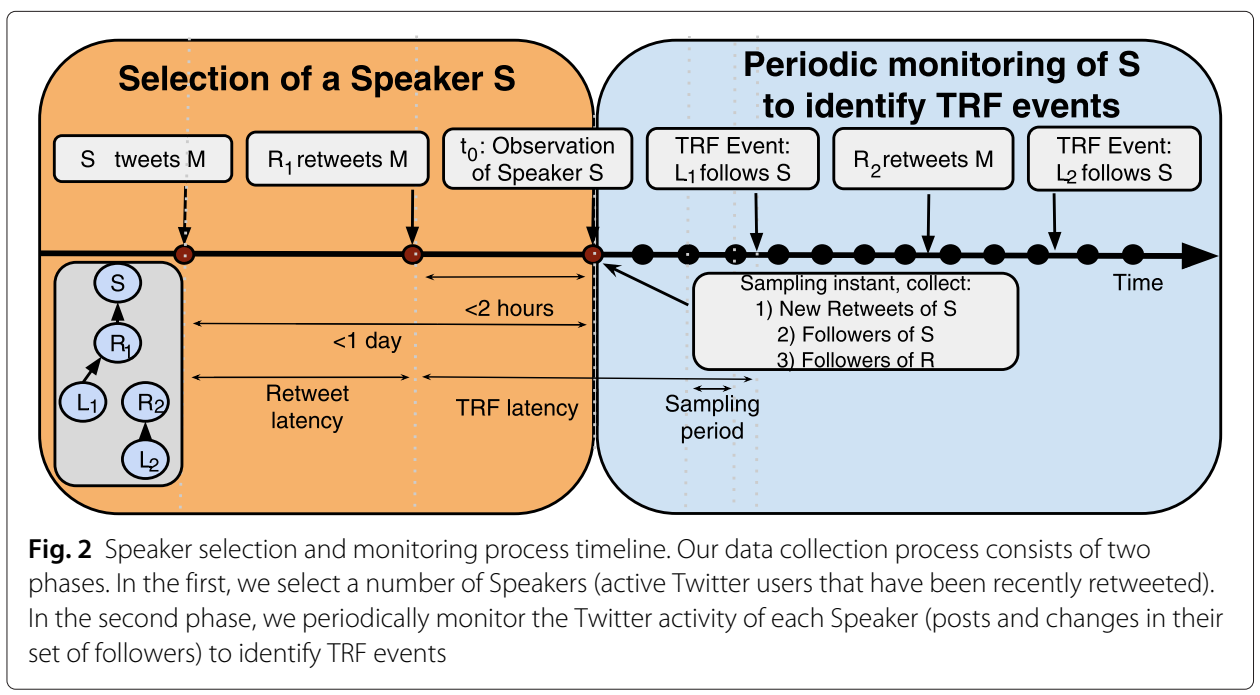


any messages [27] and that most users check their Twitter feeds rarely [28]. A random user selection process would most likely visit a number of users without recent posts, wasting a large number of our limited Twitter API calls. The adopted sampling method ensures that we monitor users that have recently posted a tweet. Specifically, we crawl the Twitter search page [29] based on a single-character search selected at random from the set of $[1-9 A-Z a-z]$. The search returns the latest 20 tweets containing the search term. We identify the users that posted these tweets and add them to our monitored Speakers set. For each selected Speaker, we also collect information about their "join time", number of followees, followers and posted tweets. For each observed tweet, we collect the time it was posted and the posted message. Note that the collected tweets are not limited to the English language (as long as they include at least one numeric digit or English character).

Given this set of monitored Speakers, we look for any retweets of their tweets posted during the last $2 \mathrm{~h}$. We only consider retweets that are flagged as such by the Twitter API. For each retweet, we retrieve the set of followers, set of followees of the Speaker and the Repeater $R$ at the time instant we first observed that retweet. Additionally, we collect the set of followers and followees of the Repeater at that time.

\section{Monitoring of Speakers}

The previous process results in a number of possible TRF events, whenever a follower of a Repeater receives a retweet of a monitored Speaker. To identify new followers, we need to examine any changes in the Speaker's followers before and after the retweet. To do so, we retrieve the set of followers of the Speaker periodically, approximately every $5 \mathrm{~min}$. We identify a TRF event when the set of followers of $S$ gains a new member (Listener $L$ ) that was previously seen in the set of followers of $R$. At that point, we log the time $L$ was seen to follow $S$ and calculate TRF latency as the time difference between the time $R$ retweeted $S$ and the time $L$ followed $S$. If $L$ received multiple retweets of $S$ (as the same tweet from multiple Repeaters, multiple tweets from the same Repeater, or multiple tweets from multiple Repeaters), we assign the TRF event to the most recent retweet of $S$ received by $L$. The intuition here is that the most recent tweets will appear at the top of $L$ 's inbox and they are more likely to be read than older retweets. At this point we also collect the set of followers and followees of the Listener.

Every $5 \mathrm{~min}$, we also update the set of monitored Speakers as follows. If a selected Speaker has not posted any tweets during the last $24 \mathrm{~h}$, we stop monitoring that user and select a new Speaker using our sampling method. The reason is that most new follower relations tend to occur within few hours from the time a Speaker has been active $[30,31]$.

\section{Data collection system}

Due to the complexity and the real-time nature of our data collection process, we need a large Twitter API request throughput. We used Twitter's API 1.0, which limits users to 350 API requests per hour. To increase this request throughput, we use a large number of distributed hosts, provided by PlanetLab, as proxies for accessing Twitter [32]. Our collection process is coordinated by a "dispatcher" application located at Georgia Tech. The dispatcher decides what data are required at any point in time and instructs a number of "workers" to request that data from Twitter. Each worker is assigned a single Planetlab host that routes API requests to Twitter. When a worker runs out of requests, it 
deactivates itself and notifies the dispatcher. At that point, the dispatcher generates a new worker, providing it with a fresh request workload.

We divide the data collection process to small independent processes, each of them requiring the smallest possible number of requests. In this way, we partition different parts of the Speaker monitoring process to a number of workers, speeding up the collection process. For instance, when requesting an update for a Speaker, the retrieval of tweets, retweets and follower sets are executed through different Planetlab hosts. Further, we limit the number of concurrently monitored Speakers to 500 to avoid overloading both Twitter and our collection system.

\section{Bot-filtering}

A major concern for any Twitter dataset is to avoid bots. Such accounts act differently than most regular Twitter users, biasing the analysis. To identify and remove bot accounts from our dataset, we revisited each account 3 months after the initial data collection to check which of those accounts have been suspended by Twitter. This practice has been used by Thomas et al. [33] as "ground truth" for the Twitter bot detection problem. Further, it has been reported that only few bots survive Twitter's policies for more than a week [34]. In our data, about $1 \%$ of the observed users were suspended by Twitter (uniformly distributed across Speakers, Repeaters, and Listeners), accounting for roughly $10 \%$ of the observed TRF events. ${ }^{2}$

\section{Collected data}

Dataset-1

To estimate the exogenous and endogenous probabilities (Section "Endogenous versus exogenous link creation") we use a small-scale dataset (compared to the dataset used in the rest of the paper). Specifically, we monitor 200 unique Twitter users (Speakers) for a period of 10 days. For each Speaker, we collect periodically (every $30 \mathrm{~min}$ ) her Twitter timeline, tweets and retweets, along with the list of her followers. We also collect the followers of every follower of the 200 monitored Speakers. Based on this dataset, we can observe all Tweet-Retweet (TR) events for every monitored Speaker over the course of 10 days, and so we can ask whether a Speaker has gained one or more new followers among the set of Listeners of her retweets.

\section{Dataset-2}

In the rest of the paper, we use a larger dataset. This dataset was collected during 1 week, from September 19 to September 25, 2012. During this time period, we collected about 300 GBytes of raw Twitter data. In this dataset we monitored 4746 Speakers that posted 386,980 tweets. These messages were retweeted 146,867 times by 83,860 distinct Repeaters. Twitter allows users that are not following a Speaker to retweet her messages. For this reason, in Dataset-2, we do not require that the Repeaters are followers of the Speaker. After removing bot accounts, we end up with 7451 observed TRF events. This figure represents $17 \%$ of the new follower links observed in our dataset.

\section{Endogenous versus exogenous link creation}

A user also gains new followers due to exogenous factors, such as Twitter's "Who to follow" service [35]. Here, we compare the likelihood with which a user gains new followers 
when there are no recent retweets of her messages (exogenous link creation) compared to the case that she gains new followers when at least one of her messages has been recently retweeted (endogenous link creation).

We focus here on potential new followers $L$ of $S$ that were already following a follower of $S$. That is, we only examine three-actor relations in which $L \rightarrow R$ and $R \rightarrow S$. We then ask "is it more likely that $L$ will follow $S(L \rightarrow S)$ when $L$ received a retweet of $S$ through $R(T R F$ event) or when $L$ did not receive any retweet from her followees that follow $S$ (TF event)?" Fig. 3a illustrates the TRF and TF events. Note that the difference between endogenous (TRF) and exogenous (TF) events is the retweet of $S$ from $R$; the local structure and the activity of $S$ remain the same in both cases.

We estimate the probability $P_{E X O}(\Delta)$ of exogenous new followers as follows. Consider a tweet of Speaker $S$ at time $t_{s}$. Suppose that this tweet is not retweeted by any of the followers of $S$ in the period $\left[t_{s}, t_{s}+\Delta\right]$. Let $\Phi\left(S, t_{s}\right)$ be the set of followers of followers of $S$ that are not directly following $S$ at $t_{s}$, i.e., $\Phi\left(S, t_{s}\right)=\left\{X: X \notin F\left(S, t_{s}\right), X \in F\left(Y, t_{s}\right), Y \in\right.$ $\left.F\left(S, t_{S}\right)\right\}$. What is the fraction of these users that follow $S$ by time $t_{s}+\Delta$ ?

$$
P_{\operatorname{EXO}}(\Delta)=\frac{\left|L: L \in \Phi\left(S, t_{s}\right), L \in F\left(S, t_{s}+\Delta\right)\right|}{\left|\Phi\left(S, t_{s}\right)\right|}
$$

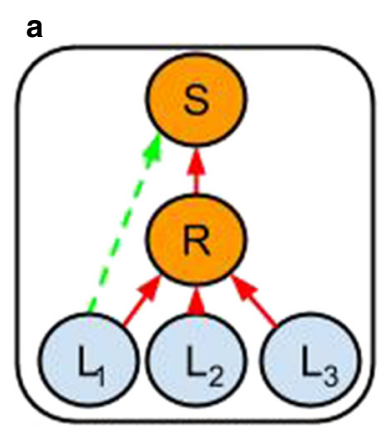

(i) TRF event: Follow after receiving a Retweet

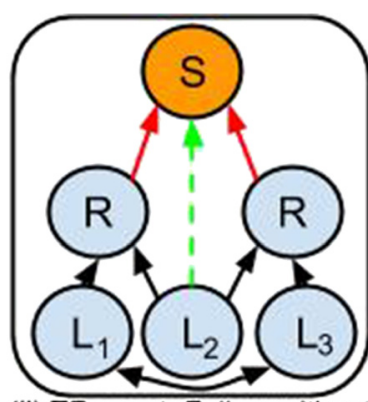

(ii) TF event: Follow without receiving a Retweet

b

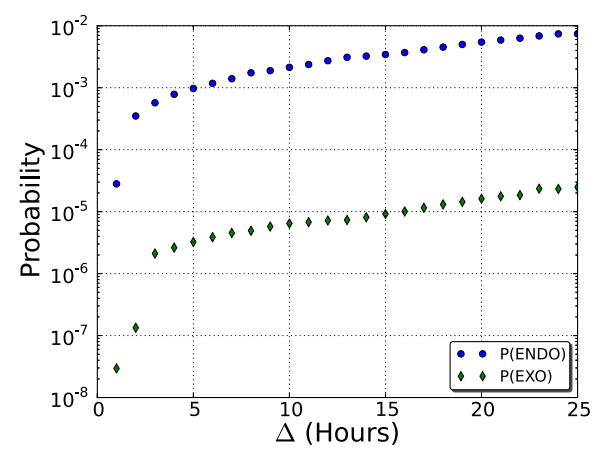

Fig. 3 a Controlling for the structural relation between $S, R$ and $L$ and for the activity of $S$ allows us to compare the likelihood of a new follower $L$ when $L$ received a retweet of $S$ (i) compared to the case that $L$ did not receive a retweet of $S$ (ii). The arrow direction shows who follows whom. Orange nodes represent tweet or retweet activity. Red edges show the extent of information propagation. Green dashed edges show new follower links. b Probability that a Speaker $S$ gains at least one new follower $L$ within an interval $\Delta$ from the time of a tweet (TF) or retweet (TRF) of $S$. The Listener $L$ is not a follower of $S$ at the time of the tweet (TF) or retweet (TRF) 
Similarly, we estimate the probability $P_{\mathrm{ENDO}}(\Delta)$ of endogenous new followers as follows. Consider again a tweet of Speaker $S$ at time $t_{s}$ but suppose that this message has been retweeted by a specific follower of $S$, referred to as Repeater $R$, at time $t_{r}>t_{s}$. Let $\Phi_{R}\left(S, t_{r}\right)$ be the subset of $\Phi\left(S, t_{r}\right)$ that includes only followers of $R$. What is the fraction of these users that follow $S$ by time $t_{r}+\Delta$ ?

$$
P_{\mathrm{ENDO}}(\Delta)=\frac{\left|L: L \in \Phi_{R}\left(S, t_{r}\right), L \in F\left(S, t_{r}+\Delta\right)\right|}{\left|\Phi_{R}\left(S, t_{r}\right)\right|}
$$

In a small-scale dataset (Dataset-1), we observed 4945 new followers for the 200 monitored Speakers over 10 days. TRF events accounted for $42 \%$ of these new links. This shows that TRF events are rather infrequent, compared to tweets and retweets, but they are responsible for a large percentage of the new links in Twitter.

Figure $3 \mathrm{~b}$ compares the two probabilities for increasing values of $\Delta$, averaged across all TF and TRF events in our dataset. We omit confidence intervals because they are too narrow. Note that the probability of endogenous new followers is consistently much higher than the probability of exogenous new followers. Especially for short $\Delta$ (up to $2 \mathrm{~h}$ ), $P_{\text {ENDO }}$ is three orders of magnitude higher than $P_{\text {EXO }}$. The difference drops to two orders of magnitude and remains stable even for values of $\Delta$ larger than $24 \mathrm{~h}$.

Please note that the previous comparison does not prove causality: we cannot be certain whether a user $L$ decided to follow $S$ because she received a retweet of $S$. However, if $L$ had not received that retweet, it would be 100-1000 times less likely that she would follow $S$ within a given time interval.

Figure $3 \mathrm{~b}$ shows that $P_{\text {ENDO }}$ increases significantly as $\Delta$ increases to about $24 \mathrm{~h}$. After that point, $P_{\mathrm{ENDO}}$ saturates to a value that is about $10^{-2}$. It can be argued that this underestimates the actual TRF probability. The reason is that a large fraction of Twitter users are either completely inactive or they do not visit Twitter often. Recent statistics report that only $20 \%$ of registered users visit Twitter at least once per month [36]. Additionally, a report from Pew Internet [37] in 2010 reported that only $36 \%$ of Twitter users check their inbox at least once a day.

\section{TRF characteristics}

The previous analysis verifies our initial intuition that the likelihood with which a user $L$ follows a user $S$ greatly increases when $L$ receives a retweet of $S$. Furthermore, this likelihood is also affected by the length of the interval between the retweet and the time $L$ observed of that retweet. We now give a more precise definition of Tweet-Retweet-Follow events. We say that a Tweet-Retweet-Follow event between users $S, R$, and $L$, where $R$ might not be a direct follower of $S$, occurs when we observe the following sequence of events:

1. $S$ tweets a message $M$ at time $t_{s}$,

2. A user $R$ retweets $M$ at some time $t_{r}>t_{s}$,

3. A user $L$, who is a follower of $R$ (i.e. $L \rightarrow R$ ) at $t_{r}$ but not a follower of $S$, follows $S$ by time $t_{l}$, where $t_{l} \in\left[t_{r}, t_{r}+\Delta\right]$.

We collected a larger dataset (Dataset-2) that we use to analyze and model TRF events. In this dataset, we observe 7451 TRF events, which represent $17 \%$ of the observed new follower relations. 


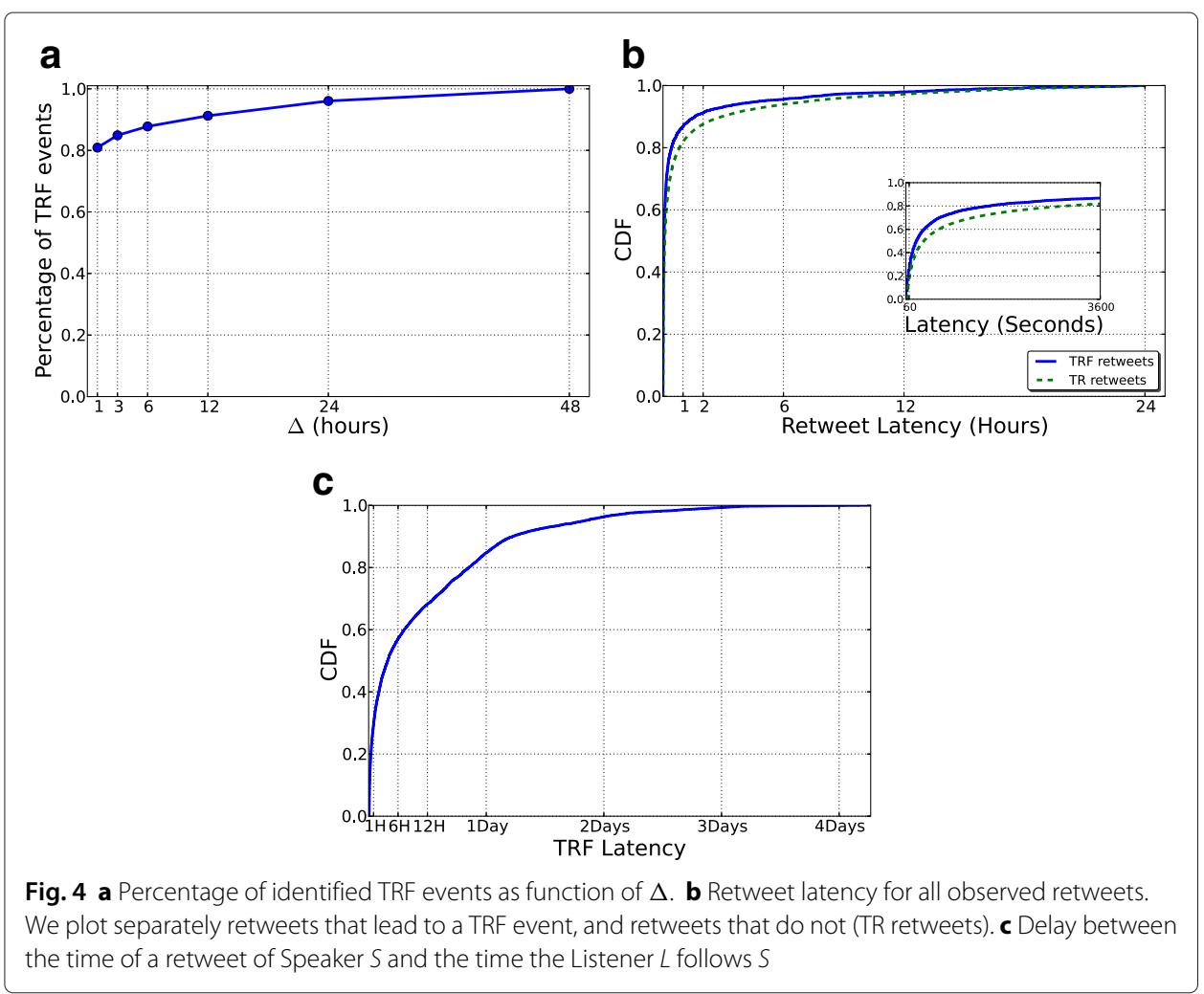

$\Delta$ is the only parameter in this definition, and it affects the likelihood of TRF events. Figure 4a shows the percentage of identified TRF events as a function of the parameter $\Delta$. As expected, the number of TRF events increases with $\Delta$ but most of them occur within $24 \mathrm{~h}$ from the corresponding retweet.

\section{Retweet latency}

Figure $4 \mathrm{~b}$ distinguishes between retweets that resulted in at least one TRF event (TRF retweets) and retweets that did not result in a TRF event (TR retweets). The analysis of these retweet events shows that more than $90 \%$ of them occur in less than an hour from the corresponding tweet; we refer to this time interval as retweet latency. This result supports the idea that "retweeting users" tend to act soon after new information becomes available.

\section{TRF latency}

We observe new $L \rightarrow S$ relations even 4 days after $L$ has received a retweet of $S$, as shown in Fig. 4c. However, more than $80 \%$ of the TRF events occur in less than $24 \mathrm{~h}$ after the retweet. Unless stated otherwise, in the rest of this paper we set $\Delta=24 \mathrm{~h}$.

\section{TRF probability}

For each monitored Speaker, we collect at each sampling instant her list of followers $F(S)$, tweets, retweets, Repeaters and the set of followers for each Repeater $F(R)$. We then identify the set of Tweet-Retweet (TR) events for each retweet of Speaker $S: T R\left(S, R, L, t_{r}, I_{\Delta}\right)$. A TR event denotes that Listener $L$ received a message of $S$ at time $t_{r}$ through a retweet 
by Repeater $R$. The indicator variable $I_{\Delta}$ is 1 if $L$ followed $S$ during a time period of length $\Delta$ after $t_{r}$.

We could define the TRF probability as the fraction of TR events for which $I_{\Delta}=1$. This calculation, however, does not consider that a Listener may receive multiple retweets (of the same or different tweets) of that Speaker. It would not be realistic to assume that the Listener will decide whether to follow the Speaker immediately after each retweet. Typically, users do not read each tweet immediately when it is generated, nor they have an infinite attention span that would allow them to consider all tweets in their inbox [31]. It is more reasonable to expect that each time a user opens her inbox she reads several recent tweets at the same time. So, we assume that a Listener decides whether to follow a Speaker based on a group of received retweets that were recently received.

Specifically, we group TR events into Retweet Groups (RG) as follows. Each RG is represented as $R G\left(S, L, t_{r}, n, I_{\Delta}\right)$, where $S$ and $L$ are the Speaker and Listener, respectively, $t_{r}$ is the timestamp of the first retweet in that group, and $n$ is the number of retweets of $S$ received by $L$ during the time window $<t_{r}, t_{r}+\Delta>$. Note that these retweets may be generated by different Repeaters. The indicator variable $I_{\Delta}$ is 1 if $L$ followed $S$ by the end of the previous time interval. If $L$ followed $S$ at time $t_{r} \leq t \leq t_{r}+\Delta$, the corresponding RG includes only those retweets received by $L$ before $t$; any subsequent retweets are ignored because $L$ already follows $S$.

Based on this Retweet Grouping method, we calculate the TRF probability $P_{\operatorname{TRF}}(\Delta)$ as the fraction of RGs for which $I_{\Delta}=1$.

\section{Factors that affect the TRF probability}

We now examine a number of factors that may affect the TRF probability. The small magnitude of the TRF probability makes the identification of important factors more challenging [38]; the following results, however, are given with satisfactory statistical significance (see $p$ values in Table 1).

Table 1 lists the structural and informational factors (features) we consider. ${ }^{3}$ We use logistic regression to analyze how these features correlate with the TRF probability. Based

Table 1 List of examined factors

\begin{tabular}{|c|c|c|c|}
\hline Factor & Description & Odds ratio & $95 \% \mathrm{Cl}$ \\
\hline \multicolumn{4}{|l|}{ Structural features } \\
\hline$|F(S)|$ & Number of followers of $S$ & $1.000^{* * *}$ & {$[1.000,1.000]$} \\
\hline$\left|F^{\prime}(S)\right|$ & Number of followees of $S$ & $0.999^{* * *}$ & {$[0.999,0.999]$} \\
\hline $\operatorname{AGE}(S)$ & Number of days since $S$ joined Twitter & $0.998^{* * *}$ & {$[0.998,0.998]$} \\
\hline$S \rightarrow L$ & $\begin{array}{l}\text { Reciprocity: whether the Speaker was following the } \\
\text { Listener at the time of the TR event }\end{array}$ & $27.344^{* * *}$ & {$[25.663,29.136]$} \\
\hline \multicolumn{4}{|l|}{ Informational features } \\
\hline$|S T(S)|$ & Total number of tweets of $S$ & $1.000^{* * *}$ & {$[1.000,1.000]$} \\
\hline$A_{\text {rate }}(S)$ & Rate of $S$ tweets per day & $0.989^{* * *}$ & {$[0.988,0.991]$} \\
\hline Tweets $(S, L, \Delta)$ & $\begin{array}{l}\text { Number of distinct tweets of } S \text { received by } L \text { during } \\
\text { period } \Delta\end{array}$ & $2.010^{* * *}$ & {$[1.781,2.270]$} \\
\hline $\operatorname{Retweets}(S, L, \Delta)$ & $\begin{array}{l}\text { Number of distinct retweets of } S \text { received by } L \text { during } \\
\text { period } \Delta\end{array}$ & $1.603^{* * *}$ & {$[1.371,1.873]$} \\
\hline $\operatorname{Repeaters}(S, L, \Delta)$ & $\begin{array}{l}\text { Number of Repeaters } R \text { that } L \text { received tweets of } S \\
\text { from during period } \Delta\end{array}$ & $2.076^{* * *}$ & {$[1.889,2.282]$} \\
\hline
\end{tabular}


on (3), we estimate the correlation coefficient $\kappa_{i}$ for each factor $X_{i} . \kappa_{i}$ denotes the effect of $X_{i}$ to the "odds" of TRF events,

$$
\ln \left(\frac{P_{\mathrm{TRF}}}{1-P_{\mathrm{TRF}}}\right)=\kappa_{0}+\sum_{i=1}^{n} \kappa_{i} X_{i}
$$

Table 1 shows the odds ratio and the corresponding $95 \%$ confidence interval for each feature. An odds ratio $\rho$ represents a $\rho \times P_{\text {TRF }}$ increase in the TRF probability for every unit increase of the corresponding feature. Thus, odds ratios close to 1 suggest that those features have no major effect on the TRF probability. Table 1 shows that all odds ratios are statistically significant $(p<0.01)$.

The "Twitter age" of the Speaker, the number of followers and followees (factors that were previously shown to correlate with Twitter activity) as well as the tweeting $[28,39]$ and retweeting [40] rate of the Speaker, show no correlation with the TRF probability. Similar results are obtained when examining the age and number of followers or followees of the Listener. We have also examined a number of aggregated informational features, namely the Speaker's overall activity and her daily tweeting activity. Both features show no significant correlation with the TRF probability.

\section{Reciprocity}

A structural feature that examines the reverse relation between $S$ and $L$, i.e., whether $S$ was already following $L$ when $L$ received one or more retweets of $S$, has a large effect on the TRF probability. Reciprocity increases the probability that $L$ will follow $S$ by 27.3 times compared to the base TRF probability. Previous work has shown reciprocity to be a dominant characteristic of several online social networks such as Twitter [28], Flickr [41], and Yahoo 360 [42].

In $44 \%$ of the observed TRF events, $S$ was following $L$ prior to the formation of the reverse link. Figure 5 shows $P_{\mathrm{TRF}}(\Delta)$ independent of reciprocity (solid line), when reciprocity is present (dashed line), and when reciprocity is not present (dotted line). When reciprocity is present, the TRF probability, denoted by $P_{\mathrm{TRF}}(\Delta, \leftrightarrow)$, is one order of magnitude larger than the probability without reciprocity, denoted by $P_{\mathrm{TRF}}(\Delta, \rightarrow)$. For $\Delta>$ $3 \mathrm{~h}, P_{\mathrm{TRF}}(\Delta, \leftrightarrow)$ further increases and gradually becomes up to two orders of magnitude larger.

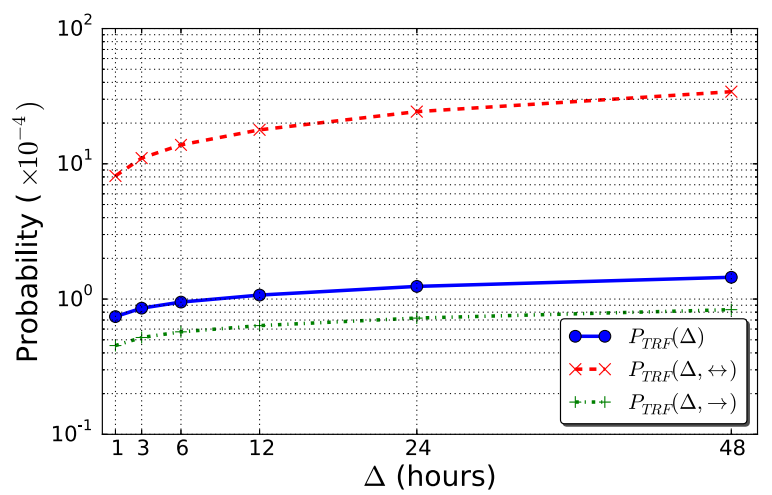

Fig. $5 P_{\mathrm{TRF}}(\Delta)$, reciprocal $P_{\mathrm{TRF}}(\Delta, \leftrightarrow)$ and non-reciprocal $P_{\mathrm{TRF}}(\Delta, \rightarrow)$ 
The large quantitative effect of reciprocity on the TRF probability implies that there may be different reasons for the formation of a link from $L$ to $S$ in that case. The existence of the reverse link, $S \rightarrow L$, could imply that these two users have some prior relation. They may know each other in other social contexts (online or offline) or they may belong to similar interest groups. In such cases, the retweet of $S$ can make $L$ aware of the existence and activity of $S$ in the Twitter network.

\section{Number of tweets and repeaters}

Earlier social influence studies showed that the probability that an individual adopts a new behavior increases with the number of her ties already engaging in that behavior $[1,17,43-45]$. Similarly, we examine whether the number of tweets and retweets of $S$ received by $L$ affects the TRF probability. It turns out that $P_{\mathrm{TRF}}$ increases with both the number of distinct tweets of $S$ that $L$ receives (odds ratio $=2.01$ ), and with the number of distinct Repeaters that $L$ received retweets from (odds ratio $=2.08$ ).

For simplicity, we choose to aggregate the number of distinct Repeaters and the number of distinct tweets of $S$ that $L$ received into a single parameter: the total number $n$ of retweets (potentially not distinct) of $S$ that were received by $L$ in a time period of length $\Delta$. This new factor has high correlation with the TRF probability (odds ratio $=1.25$, $p<0.001)$. Figure 6 (left) shows the TRF probability in the absence of reciprocity $(L \rightarrow S)$ while Fig. 6 (right) shows the TRF probability in the presence of reciprocity $(L \leftrightarrow S)$, as a function of $n$.

\section{TRF model}

We now construct a simple model for the probability of TRF events. The objective of this exercise is to create a parsimonious probabilistic model that can be used in analytical or computational studies of co-evolutionary dynamics in social networks.

The model considers two independent mechanisms behind each TRF event: How many retweets $n$ of Speaker $S$ did the Listener $L$ receive? And, $\operatorname{did} L$ actually observe (i.e., read) this group of retweets? The simplest approach is to assume, first, that the $n$ received retweets are either observed as a group with probability $p$ or they are completely missed, and second, that each observed retweet causes a TRF event independently and with the same probability $q$. Then, the probability of a TRF event after receiving at most $n$ retweets is

$$
P_{\mathrm{TRF}}(n)=p \times\left(1-(1-q)^{n}\right)
$$
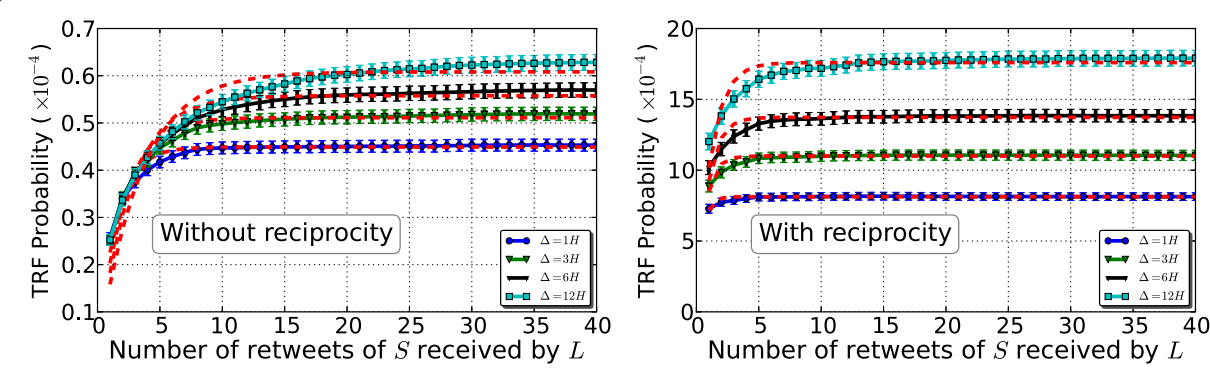

Fig. 6 Empirical (solid) and model-based (dashed) TRF probability $P_{\operatorname{TRF}}(\rightarrow, n)$ (left) and $P_{\operatorname{TRF}}(\leftrightarrow, n)$ (right) as a function of the number $n$ of received retweets of $S$ at $L$, for four different values of $\Delta$ 
Table 2 Estimated value of the two model parameters $p$ and $p \times q$

\begin{tabular}{lllll}
\hline$\Delta(\mathrm{h})$ & $p$ & $p \times q$ & $p$ & $p \times q$ \\
& Without reciprocity & & With reciprocity & \\
\hline 1 & $0.5 \times 10^{-4}$ & $0.12 \times 10^{-4}$ & $8.1 \times 10^{-4}$ & $7.2 \times 10^{-4}$ \\
3 & $0.5 \times 10^{-4}$ & $0.13 \times 10^{-4}$ & $11.0 \times 10^{-4}$ & $8.5 \times 10^{-4}$ \\
6 & $0.6 \times 10^{-4}$ & $0.14 \times 10^{-4}$ & $13.0 \times 10^{-4}$ & $9.3 \times 10^{-4}$ \\
12 & $0.6 \times 10^{-4}$ & $0.15 \times 10^{-4}$ & $17.6 \times 10^{-4}$ & $9.3 \times 10^{-4}$ \\
24 & $0.7 \times 10^{-4}$ & $0.16 \times 10^{-4}$ & $24.0 \times 10^{-4}$ & $10.2 \times 10^{-4}$ \\
48 & $0.8 \times 10^{-4}$ & $0.16 \times 10^{-4}$ & $33.1 \times 10^{-4}$ & $10.2 \times 10^{-4}$ \\
\hline
\end{tabular}

Thus, the probability of a TRF event after only one received retweet is $p \times q$. For a large number of received retweets, the TRF probability tends to the observation probability $p$.

As shown in Fig. 6 (left), the measured TRF probability $P_{\mathrm{TRF}}(\rightarrow, n)$ without reciprocity seems to "saturate" after $n$ exceeds about 10-20 retweets. The same trend is observed in the case of reciprocity (Fig. 6 (right)), but the saturation appears earlier (after around $5-10$ retweets). The model of (4) captures the dependency with $n$ quite well. The parameters $p$ and $q$ depend on reciprocity as well as on the time window $\Delta$, as shown in Table 2 . Reciprocity increases significantly both the observation probability $p$ and the probability $p \times q$ that a single received retweet will cause a TRF event. As expected, increasing the observation time window $\Delta$ increases the observation probability. The effect of $\Delta$ on the probability $p \times q$ is weaker, especially when there is no reciprocity.

We further examined the accuracy of the proposed model through a cross-validation approach. We split the dataset in two equal parts, one for parameterizing the model and another for testing that model. Figure 7 shows this comparison for different values of $\Delta$.

\section{Implications of TRF events}

Most prior work in online social networks focused either on the exogenous evolution of the topology (dynamics of networks) or on influence and information diffusion on static networks (dynamics on networks), ignoring the potential coupling between these two dynamics. We now discuss how TRF events may gradually transform the structure of a social network. We consider two fundamentally different network topologies, and discuss the implications of TRF events from the information diffusion perspective.

\section{Effect on topologies with directed cycles}

The left graph of Fig. 8a shows a weakly connected network, which may be a subset of the Twitter topology. A directed cycle exists between some of its nodes, namely $A \rightarrow B \rightarrow$
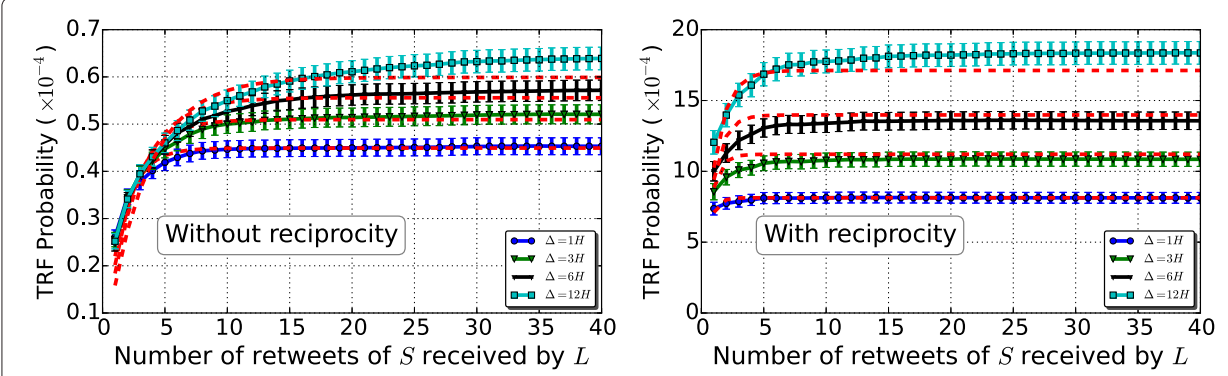

Fig. 7 Cross-validation of the proposed model. Half of the observed TRF events are used to parameterize the model of Eq. 4 (dashed lines), while the other half is used to estimate the TRF probability empirically (solid lines) 


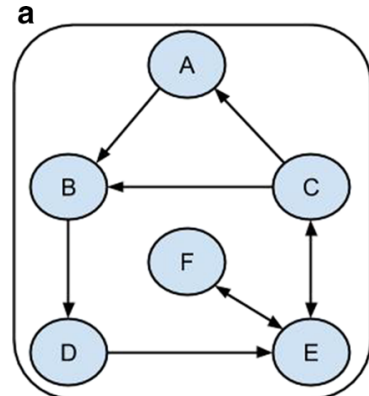

(i) Initial network topology

b

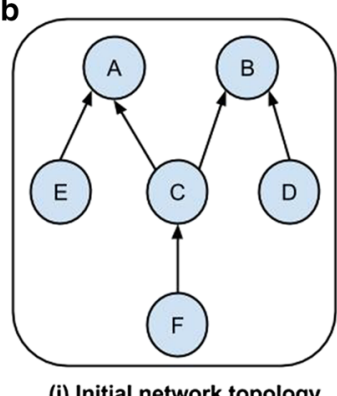

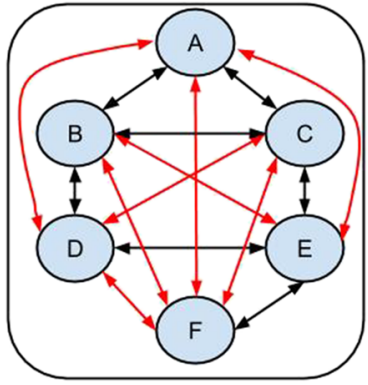

(ii) Final network topology

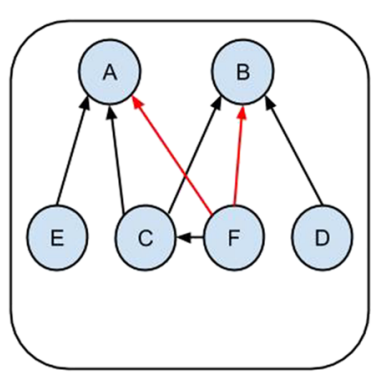

(ii) Final network topology

Fig. 8 a An initial network that includes a directed cycle. A sequence of TRF events can transform this cycle to a clique, meaning that the corresponding users gradually form a tightly knit community. $\mathbf{b}$ A hierarchical initial network. A sequence of TRF events can transform this multi-layer hierarchy into a two-layer hierarchy in which each sink node is directly followed by a set of other nodes (its "sphere of influence"), while each non-sink node follows at least one sink node

$D \rightarrow E \leftrightarrow C \rightarrow A$. Let us focus on the largest directed cycle in this network, i.e., in its largest strongly connected component (SCC). The ties of the participating nodes may also include links to or from nodes out of this cycle, such as the $E \leftrightarrow F$ relation.

Suppose that $A$ posts a tweet at some point in time and $C$ decides to retweet it. Node $E$ will receive that retweet and may follow $A$ (TRF event). It is easy to see that, after a sufficiently large number of TRF events, the nodes of this directed cycle will form a fully connected directed graph, as shown in the right graph of Fig. 8a (red edges denote connections created through TRF events), in which everyone is following all others. This transformation can only take place when a cycle already exists in the initial network; TRF events cannot create directed cycles. So, when an initial network includes a directed cycle, a sequence of TRF events may transform that cycle into a clique in which everyone can generate information that all others receive directly from the source.

\section{Effect on hierarchical topologies}

The left graph of Fig. 8b shows a hierarchical weakly connected directed network. Again, this network may be a subset of the Twitter topology. This network contains no directed cycles, but a number of sink nodes (i.e., nodes with no outgoing edges; $A$ and $B$ in this example).

User $F$ may receive a retweet of $A$ and $B$ through $C$, and she may then decide to follow them. After a sequence of TRF events, this network can then reach the topological equilibrium shown in the right graph of Fig. 8b, in which no new links can be added through 
TRF events. More generally, suppose that $F^{\prime}(X)=\left\{X_{1}, \ldots, X_{n}\right\}$ is the set of followees of $X$. The set of Speakers that $X$ may receive a retweet from can be defined recursively as $F_{U}^{\prime}(X)=F^{\prime}(X) \cup\left(F_{U}^{\prime}\left(X_{1}\right) \cup \ldots F_{U}^{\prime}\left(X_{n}\right)\right)$; if user $X$ does not have any followees then $F_{U}^{\prime}(X)$ is the empty set. It is easy to see that, after a sufficiently large number of TRF events, a multi-layer hierarchical network will converge to a two-layer hierarchy in which every non-sink user $X$ follows all users in $F_{U}^{\prime}(X)$. Then, an initial sink node $X$ will be followed directly by all users that had a directed path towards $X$ in the initial network. A consequence of TRF events in such hierarchical networks is the emergence of some highly influential users that were the sink nodes in the initial network. Further, non-sink nodes will be partitioned, with the users in each partition following a distinct set of sink nodes.

The previous two topologies are obvious extremes. In practice, a given weakly connected subset of Twitter users may contain groups of nodes that form directed cycles as well as nodes that do not belong in any directed cycle. An interesting question then is: given a weakly connected directed social network, what fraction of its nodes belong to the longest directed cycle (i.e., largest SCC) in that network? If this fraction is large, the network resembles the example of Fig. 8a, while if it is close to zero the network is similar to the example of Fig. 8b.

We investigated the previous question based on samples of the actual Twitter topology, at least as it was measured by Kwak et al. [28] in $2010 .{ }^{4}$

We collected weakly connected network samples using the Random-Walk [46] and Snowball (Breadth-First-Search) [47] sampling methods. The largest SCC was determined with Tarjan's algorithm [48].

In the case of moderately large samples, between 1000 to 1,000,000 nodes, the largest SCC contained consistently more than $90 \%$ of the nodes. This result suggests that the Twitter topology is closer to the network of Fig. 8a than to the network of Fig. 8b. The creation of such large cliques, however, may require a very long time, and it may also be impractical for a user to follow thousands of other users. Consequently, we are more interested in smaller samples, including only tens or hundreds of Twitter users.

Figure 9 shows the percentage of Twitter users that are included in the SCC of small network samples, in the range of $10-1000$ nodes. Each point is the average

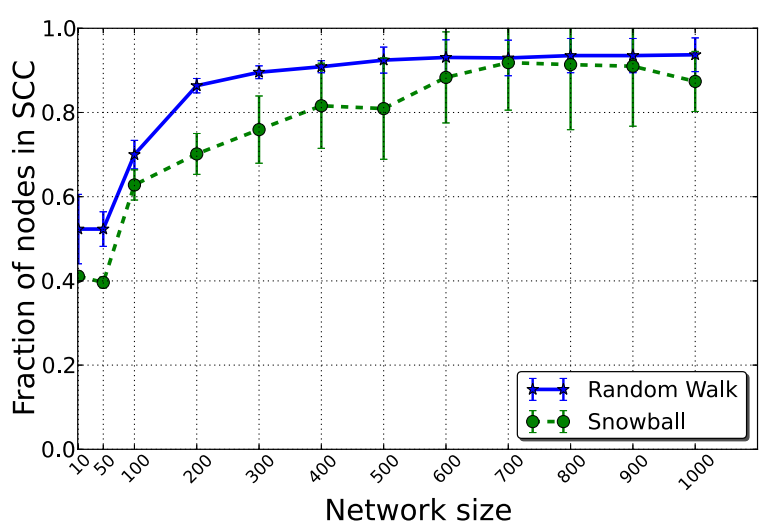

Fig. 9 Fraction of Twitter nodes in the largest SCC for different sample sizes, using two sampling methods 
of 1000 samples of that size, and the error bars represent $95 \%$ confidence intervals. Independent of the sampling method, the SCC typically includes the majority of the nodes even for samples of few tens of users. The SCC percentage increases to about $80-90 \%$ for networks with more than $200-400$ users. These results imply that co-evolutionary dynamics, and the TRF mechanism in particular, have the potential to gradually create very dense communities of users in which everyone is following almost everyone else, as long as the involved users are active, tweeting and retweeting information.

\section{Unfollow events}

TRF events can be considered as only one instance of co-evolutionary dynamics in social networks. More such mechanisms may exist however. For instance, a sequence of one or more tweets from a Speaker $S$ received by a follower $L$ may cause $L$ to remove the link to $S$; we refer to this as an endogenous unfollow event. On the other hand, exogenous unfollow events occur when $L$ removes the link to $S$ for reasons that are unrelated to $S$ 's tweeting activity. In the rest of this section, we briefly investigate unfollow events. Unfortunately, we are not able to distinguish between endogenous and exogenous unfollow events. Instead, we simply examine the timing of unfollow events relative to the Speaker's last tweet and analyze statistically the effect of various structural and informational features on the probability of unfollow events.

Kwak et al. showed through data analysis and user interviews that unfollow events are highly correlated with the tweeting activity of the Speaker [49]. Additionally, KivranSwaine et al. [50] showed that structural properties of two individuals significantly affect the probability that they will be connected in the future.

\section{Unfollow data}

We monitor a set of Speakers selected as described in Section "Data collection". One difference is that we collect periodically only the set of followers of $S$; we do not collect retweets, repeaters and their followers. A follower $L$ of $S$ is said to unfollow $S$ at a sampling instant $t_{k+1}$ if $L$ is in $F\left(S, t_{k}\right)$ but not in $F\left(S, t_{k+1}\right)$. As in the case of TRF events, the sampling period is about $5 \mathrm{~min}$.

Additionally, we download the total activity of each monitored Speaker during the data collection period (1 week). This activity includes the original tweets posted by the Speaker as well as tweets of others that were retweeted by the Speaker. We also log the time of the tweet or retweet, and the initiator of that post in the case of a retweet.

This "unfollow dataset" includes 3648 monitored Speakers, while the initial number of followers (before any unfollow events) is 4,055,327 (3,609,649 distinct users). During the 1-week data collection period, we observed 5325 unfollow events $(0.13 \%$ of the total number of followers)) from 5220 Listeners to 983 Speakers.

Figure 10 shows the CDF of the latency between the time $L$ unfollowed $S(L \nrightarrow \rightarrow S)$ and the last activity of $S$ received by $L$ before the unfollow event. Almost $60 \%$ of the unfollow events occur during the first hour after $S$ has posted some content, and almost $100 \%$ of the unfollow events occur within a day. This observation suggests that many unfollow events may be endogenous. We cannot distinguish between endogenous and exogenous unfollow events strictly based on this latency, however, especially when the Speaker tweets at a high frequency (say, several times per day). 


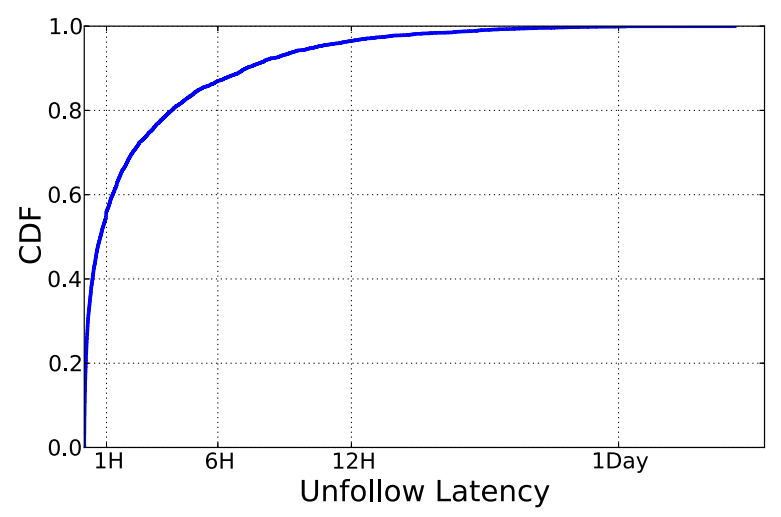

Fig. 10 Elapsed time between the last activity of S observed by $L$ and the time of the unfollow event $L \nrightarrow S$

\section{Unfollow probability}

How likely is for a Listener $L$ to unfollow Speaker $S$ during a time period $\Delta$ after receiving a tweet (or retweet) from $S$ ? We define the probability of an unfollow event similar to the TRF probability. We first identify all Activity events (A) for each post of each Speaker $S$. An Activity event is denoted by $A\left(S, L, t_{a}, I_{R}, I_{\Delta}\right)$ and it means that follower $L$ of $S(L \in F(S))$ received a tweet or retweet from $S$ at time $t_{a}$. The indicator variable $I_{R}$ is 1 if the message was a retweet, and 0 if it was an original tweet of $S$. The indicator variable $I_{\Delta}$ is 1 if $L$ unfollowed $S$ during a time period of length $\Delta$ after $t_{a}$.

We group such Activity events to Activity Groups (AG) of the form AG(S, $L, t_{a}$, $\left.n, n_{t}, n_{r}, I_{\Delta}\right) . n, n_{t}, n_{r}$ denote the total number of posts, tweets, and retweets of $S$ received by $L$ during the time window $\left\langle t_{a}, t_{a}+\Delta>\right.$. The grouping method is similar to the clustering of TR events in RG. We then calculate the probability of an unfollow event $P_{\mathrm{UNF}}(\Delta)$ as the fraction of AGs for which $I_{\Delta}=1$. Figure 11 shows the unfollow probability $P_{\mathrm{UNF}}$ as a function of $\Delta$.

Using the multivariate logistic regression model of Eq. 3, we estimate the correlation between a number of features and the unfollow probability. We use features similar to those described in Table 1, but excluding any Repeater-related features. The "number

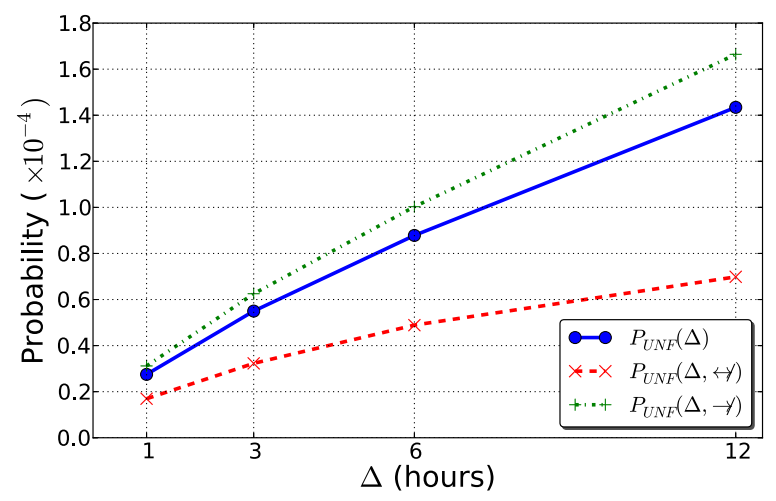

Fig. 11 Overall $\left(P_{\mathrm{UNF}}(\Delta)\right)$, reciprocal $\left(P_{\mathrm{UNF}}(\Delta, \Varangle)\right)$ and non-reciprocal $\left(P_{\mathrm{UNF}}(\Delta, \nrightarrow)\right)$ unfollow probability as a function of $\Delta$ 
Table 3 Odds ratio and its $95 \%$ confidence interval for each feature of the multivariate logistic regression model for $P$ UNF

\begin{tabular}{|c|c|c|}
\hline & Odds ratio & $95 \% \mathrm{Cl}$ \\
\hline \multicolumn{3}{|l|}{ Structural features } \\
\hline$|F(S)|$ & $0.999^{* * *}$ & {$[0.999,0.999]$} \\
\hline$\left|F^{\prime}(S)\right|$ & $1.000^{* * *}$ & {$[1.000,1.000]$} \\
\hline $\operatorname{AGE}(S)$ & $0.998^{* * *}$ & {$[0.998,0.998]$} \\
\hline$S \rightarrow L$ & $0.302^{* * *}$ & {$[0.261,0.348]$} \\
\hline \multicolumn{3}{|c|}{ Informational features } \\
\hline ST(S) & $1.000^{* * *}$ & {$[1.000,1.000]$} \\
\hline$A_{\text {rate }}(S)$ & $0.972^{* * *}$ & {$[0.967,0.978]$} \\
\hline Tweets $(S, L, \Delta)$ & $1.041^{* * *}$ & {$[1.025,1.057]$} \\
\hline Retweets $(S, L, \Delta)$ & 1.026 & {$[0.992,1.006]$} \\
\hline
\end{tabular}

of tweets" and "number of retweets" refer to the number of original posts by $S$ and the number of posts forwarded by $S$, respectively.

Table 3 shows the resulting odds ratios and the corresponding $95 \%$ confidence intervals for each feature. Note that most of the features have limited or no effect on the unfollow probability; most of the structural features return an odds value close to 1 . As the number of tweets increases, $P_{\mathrm{UNF}}$ slightly increases, implying that unfollow events may be more likely for Speakers that tweet too frequently. However, this effect is not sufficiently strong.

Only the reciprocity factor seems to significantly affect $P_{\mathrm{UNF}}$. In the presence of reciprocity, meaning that the Speaker $S$ follows the Listener $L$, it is about $70 \%$ less likely for $L$ to unfollow $S$. In only $18 \%$ of the observed unfollow events $S$ followed $L$. Figure 11 shows $P_{\mathrm{UNF}}$ conditioned on the presence of reciprocity $\left(P_{\mathrm{UNF}}(\Delta, \leftrightarrow)\right)$ or conditioned on the absence of reciprocity $\left(P_{\mathrm{UNF}}(\Delta, \not \rightarrow)\right)$. Note that it is at least twice more likely for $L$ to unfollow $S$ when their relationship is not reciprocal. As discussed in the case of TRF events, reciprocal relations may represent a connection between two users outside the context of Twitter, or a stronger degree of homophily between them.

The small percentage of unfollow events in reciprocal relations may be explained as follows: Kwak et al. [49] claim that some users follow back all new followers as a courtesy. After a while, however, the former may decide that they are not interested in the posts of their new followers and unfollow them.

Kwak et al. showed that people often appreciate receiving acknowledgments from other users (in the form of replies or tweets of the same content/hashtag). Such activity often decreases the likelihood of unfollow events $[51,52]$. Hutto et al. have found that the content of someone's tweets significantly impacts the number of followers of that user [53]. Their results show that expressing negative sentiment has an adverse effect on the follower count, whereas expressing positive sentiment helps to increase the latter. This prior work has focused on a small number of snapshots that are few months apart. We plan to leverage our near real-time data collection system to monitor unfollow events and their dependence on the actual content of tweets in smaller time scales.

\section{Conclusions}

Most prior work in online social networks focused either on the exogenous evolution of the topology (dynamics of network) or on influence and information diffusion on static 
networks (dynamics on network), ignoring the potential coupling between these two dynamics. In this paper, we considered co-evolutionary dynamics in the specific case of the Twitter online social network. Most of our study focused on the addition of new links through the so-called Tweet-Retweet-Follow events. We showed that it is much more likely for a user to get a new follower if her tweets are retweeted than in the case where her tweets are not retweeted. We showed that TRF events, although infrequent compared to tweets or retweets, occur in practice and they are responsible for a significant fraction (about $20 \%$ ) of the new edges in Twitter. Through (near) real-time monitoring of many Twitter users, we showed how to identify TRF events and investigated their temporal and statistical characteristics. More than $80 \%$ of TRF events occur in less than 24 $\mathrm{h}$ after the corresponding retweet. The main factors that affect the probability of a TRF event are reciprocity and the total number of retweets received by the Listener. Based on these findings, we have proposed a simple probabilistic model for the probability of TRF events. We have also discussed how TRF events can affect the structure of the underlying social network. TRF events tend to transform directed cycles into cliques, creating closely knit communities of users in which everyone is following everyone else. The analysis of samples from the 2010 Twitter topology shows that weakly connected groups of more than 200-400 users contain large directed cycles that include more than $80-90 \%$ of the users. Finally, we have argued that TRF events are not the only form of co-evolutionary dynamics in Twitter. Users may also break existing relations (unfollow others) based on the tweeting activity of the latter. An analysis of this effect shows that $60 \%$ of the unfollow events occur during the first hour after the Speaker has posted some content. Also, a reciprocal relation (a link from the Speaker to the Listener) greatly decreases the likelihood of an unfollow event in the opposite direction. In future work, we plan to explore additional types of co-evolutionary dynamics and to quantify their effect. Such events include the creation of new follower relations after a "reply" or "mention" user action.

\section{Endnotes}

${ }^{1}$ http://en.wikipedia.org/wiki/Yahoo!_Meme

${ }^{2}$ Exploring the impact of bots on the evolution of the Twitter topology is an interesting area for future work.

${ }^{3}$ Features such as the number of common friends between $\mathrm{S}$ and $\mathrm{L}$ are not examined because they would require additional data that we have not collected.

${ }^{4}$ http://an.kaist.ac.kr/traces/WWW2010.html

Competing interests

The authors declare that they have no competing interests.

Authors' contributions

Both authors designed the research, data collection and experiments. Demetris Antoniades carried out the data collection and executed the experiments. Both authors contributed to the writing of the manuscript. Both authors read and approved the final manuscript.

\section{Acknowledgements}

This material is based upon work supported in part by the Defense Advanced Research Projects Agency (DARPA) under Contract No. W91 1NF-12-1-0043. Any opinions, findings, and conclusions or recommendations expressed in this material are those of the authors and do not necessarily reflect the views of DARPA or the U.S. Government. The views and conclusions contained in this document are those of the authors and should not be interpreted as representing the official policies, either expressly or implied, of the Defense Advanced Research Projects Agency or the U.S. Government.

Author details

${ }^{1}$ Department of Computer Science, University of Cyprus, Nicosia, Cyprus. ${ }^{2}$ College of Computing, Georgia Institute of Technology, Atlanta, Georgia, USA. 
Received: 11 February 2015 Accepted: 26 June 2015

Published online: 31 July 2015

\section{References}

1. Bakshy, E, Karrer, B, Adamic, LA: Social influence and the diffusion of user-created content. In: Proc. of the tenth ACM conference on Electronic commerce, pp. 325-334, (2009)

2. Vespignani, A: Modelling dynamical processes in complex socio-technical systems. Nat. Physics. 8(1), 32-39 (2011)

3. Leskovec, J, Kleinberg, J, Faloutsos, C: Graphs over time: densification laws, shrinking diameters and possible explanations. In: Proc. of the eleventh ACM SIGKDD international conference on Knowledge discovery in data mining, pp. 177-187. ACM, New York, NY, USA, (2005)

4. Gross, T, Blasius, B: Adaptive coevolutionary networks: a review. J. R. Society Interface. 5(20), 259-271 (2008)

5. Leskovec, J, McGlohon, M, Faloutsos, C, Glance, NS, Hurst, M: Patterns of cascading behavior in large blog graphs. In: Proc. of SIAM SDM 2007. SIAM, (2007)

6. Antoniades, D, Dovrolis, C: Co-evolutionary dynamics in social networks: A case study of Twitter. In: Proc. of the Third IEEE International Workshop on Complex Networks and their Applications, (2014)

7. Barabási, A-L, Albert, R: Emergence of scaling in random networks. Science. 286(5439), 509-512 (1999)

8. Hopcroft, J, Lou, T, Tang, J: Who will follow you back?: reciprocal relationship prediction. In: Proc. of the 20th ACM international conference on Information and knowledge management, pp. 1137-1146. ACM, (2011)

9. Papadopoulos, F, Kitsak, M, Serrano, MÁ, Boguñá, M, Krioukov, D: Popularity versus similarity in growing networks. Nature. 489(7417), 537-540 (2012)

10. Golder, SA, Yardi, S: Structural predictors of tie formation in Twitter: Transitivity and mutuality. In: Social Computing (SocialCom), 2010 IEEE Second International Conference on, pp. 88-95. IEEE, (2010)

11. Leskovec, J, Backstrom, L, Kumar, R, Tomkins, A: Microscopic evolution of social networks. In: Proc. of the 14th ACM SIGKDD International Conference on Knowledge Discovery and Data Mining, pp. 462-470, (2008)

12. Romero, DM, Kleinberg, J: The directed closure process in hybrid social-information networks, with an analysis of link formation on Twitter. In: Proc. of the 4th International AAAI Conference on Weblogs and Social Media, pp. 138-145, (2010)

13. Muchnik, L, Pei, S, Parra, LC, Reis, SD, Jr. Andrade, JS, Havlin, S, Makse, HA: Origins of power-law degree distribution in the heterogeneity of human activity in social networks. Scientific reports. 3 (2013)

14. Gallos, LK, Rybski, D, Liljeros, F, Havlin, S, Makse, HA: How people interact in evolving online affiliation networks. Phys. Rev. X. 2, 031014 (2012)

15. Lotan, G, Graeff, E, Ananny, M, Gaffney, D, Pearce, I, Boyd, D: The revolutions were tweeted: Information flows during the Tunisian and Egyptian revolutions. Int. J. Commun. 5, 1375-1405 (2011)

16. Starbird, K, Palen, L: (How) will the revolution be retweeted?: information diffusion and the 2011 Egyptian uprising. In: Proc. of the acm 2012 conference on computer supported cooperative work, pp. 7-16. ACM, (2012)

17. Romero, DM, Meeder, B, Kleinberg, JM: Differences in the mechanics of information diffusion across topics: idioms, political hashtags, and complex contagion on Twitter. In: Proc. of the 20th International Conference on World Wide Web, pp. 695-704, (2011)

18. Myers, SA, Zhu, C, Leskovec, J: Information diffusion and external influence in networks. In: Proc. of the 18th ACM SIGKDD international conference on Knowledge discovery and data mining, pp. 33-41. ACM, (2012)

19. Kozma, B, Barrat, A: Consensus formation on adaptive networks. Physical Review E. 77(1), 016102 (2008)

20. Shaw, LB, Schwartz, IB: Enhanced vaccine control of epidemics in adaptive networks. Phys. Rev. E. 81, 046120 (2010)

21. Volz, E, Meyers, LA: Epidemic thresholds in dynamic contact networks. J. R. Soc. Inter. 6(32), 233-241 (2009)

22. Rocha, LE, Liljeros, F, Holme, P: Simulated epidemics in an empirical spatiotemporal network of 50,185 sexual contacts. PLoS Comput. Biol. 7(3), e1001109 (2011)

23. Perra, N, Baronchelli, A, Mocanu, D, Gonçalves, B, Pastor-Satorras, R, Vespignani, A: Random walks and search in time-varying networks. Phys. Rev. Lett. 109, 238701 (2012)

24. Weng, L, Ratkiewicz, J, Perra, N, Gonçalves, B, Castillo, C, Bonchi, F, Schifanella, R, Menczer, F, Flammini, A: The role of information diffusion in the evolution of social networks. In: Proc. of the 19th ACM SIGKDD international conference on Knowledge discovery and data mining KDD '13, pp. 356-364. ACM, New York, NY, USA, (2013)

25. Myers, SA, Leskovec, J: The bursty dynamics of the Twitter information network. In: Proc. of the 23rd international conference on World wide web, pp. 913-924. International World Wide Web Conferences Steering Committee, (2014)

26. Meeder, B, Karrer, B, Sayedi, A, Ravi, R, Borgs, C, Chayes, J: We know who you followed last summer: inferring social link creation times in Twitter. In: Proc. of the 20th international conference on World wide web, pp. 517-526. ACM, (2011)

27. An Exhaustive Study of Twitter Users Across the World (2012). http://www.beevolve.com/twitter-statistics/. [Online; accessed 30-Jan-2014]

28. Kwak, H, Lee, C, Park, H, Moon, S: What is Twitter, a social network or a news media? In: Proc. of the 19th International Conference on World Wide Web, pp. 591-600, (2010)

29. Twitter search. http://search.twitter.com. [Online; accessed 30-Jan-2014]

30. Antoniades, D, Polakis, I, Kontaxis, G, Athanasopoulos, E, loannidis, S, Markatos, EP, Karagiannis, T: we. b: The web of short URLs. In: Proc. of the 20th international conference on World wide web, pp. 715-724. ACM, (2011)

31. Weng, L, Flammini, A, Vespignani, A, Menczer, F: Competition among memes in a world with limited attention. Sci. Rep. 2 (2012)

32. Chun, B, Culler, D, Roscoe, T, Bavier, A, Peterson, L, Wawrzoniak, M, Bowman, M: Planetlab: an overlay testbed for broad-coverage services. ACM. SIGCOMM. CCR. 33(3), 3-12 (2003)

33. Thomas, K, Grier, C, Song, D, Paxson, V: Suspended accounts in retrospect: An analysis of Twitter spam. In: Proc. of the 2011 ACM SIGCOMM conference on Internet measurement conference, pp. 243-258. ACM, (2011)

34. Sridharan, V, Shankar, V, Gupta, M: Twitter games: how successful spammers pick targets. In: Proc. of the 28th Annual Computer Security Applications Conference, pp. 389-398. ACM, (2012) 
35. Gupta, P, Goel, A, Lin, J, Sharma, A, Wang, D, Zadeh, R: WTF: The who to follow service at Twitter. In: Proc. of the 22nd international conference on World Wide Web, pp. 505-514. International World Wide Web Conferences Steering Committee, (2013)

36. statisticbrain.com: Twitter Statistics (2013). http://www.statisticbrain.com/twitter-statistics/

37. Aaron, S, Lee, R: $8 \%$ of online Americans use Twitter (2010). http://www.pewinternet.org/Reports/2010/TwitterUpdate-2010.aspx

38. He, H, Garcia, EA: Learning from imbalanced data. Knowl. Data Eng. IEEE Trans. 21(9), 1263-1284 (2009)

39. Huberman, B, Romero, D, Wu, F: Social networks that matter: Twitter under the microscope (2008). Available at SSRN: http://ssrn.com/abstract=1313405 or http://dx.doi.org/10.2139/ssrn.1313405

40. Suh, B, Hong, L, Pirolli, P, Chi, E: Want to be retweeted? large scale analytics on factors impacting retweet in Twitter network. In: Social Computing (SocialCom), 2010 IEEE Second International Conference on, pp. 177-184. IEEE, (2010)

41. Cha, M, Mislove, A, Gummadi, KP: A measurement driven analysis of information propagation in the Flickr social network. In: Proc. of the 18th international conference on World wide web, pp. 721-730. ACM, (2009)

42. Kumar, R, Novak, J, Tomkins, A: Structure and Evolution of Online Social Networks. In: Yu, PS, Han, J, Faloutsos, C (eds.) Link Mining: Models, Algorithms, and Applications, pp. 337-357. Springer, New York, (2010)

43. Backstrom, L, Huttenlocher, D, Kleinberg, J, Lan, X: Group formation in large social networks: membership, growth, and evolution. In: Proc. of the 12th ACM SIGKDD international conference on Knowledge discovery and data mining, pp. 44-54. ACM, (2006)

44. Hodas, NO, Lerman, K: How visibility and divided attention constrain social contagion. In: Proc. of the 2012 ASE/IEEE International Conference on Social Computing and 2012 ASE/IEEE International Conference on Privacy, Security, Risk and Trust. IEEE Computer Society, (2012)

45. Feng, L, Hu, Y, Li, B, Stanley, HE, Havlin, S, Braunstein, LA: Competing for Attention in Social Media under Information Overload Conditions. PLoS ONE. 10(7), e0126090 (2015)

46. Leskovec, J, Faloutsos, C: Sampling from large graphs. In: Proc. of the 12th ACM SIGKDD international conference on Knowledge discovery and data mining KDD '06, pp. 631-636. ACM, New York, NY, USA, (2006)

47. Goodman, LA: Snowball sampling. Annals Math. Stat. 32(1), 148-170 (1961)

48. Tarjan, R: Depth-first search and linear graph algorithms. SIAM Journal Comput. 1(2), 146-160 (1972)

49. Kwak, H, Chun, H, Moon, S: Fragile online relationship: a first look at unfollow dynamics in Twitter. In: Proc. of the 2011 annual conference on Human factors in computing systems CHI '13, pp. 1091-1100. ACM, (2011)

50. Kivran-Swaine, F, Govindan, P, Naaman, M: The impact of network structure on breaking ties in online social networks: unfollowing on Twitter. In: Proc. of the SIGCHI Conference on Human Factors in Computing Systems CHI '11, pp. 1101-1104. ACM, New York, NY, USA, (2011)

51. Kwak, H, Moon, S, Lee, W: More of a receiver than a giver: Why do people unfollow in Twitter? In: Proc. of AAAl ICWSM 2012, (2012)

52. $\mathrm{Xu}, \mathrm{B}$, Huang, Y, Kwak, H, Contractor, N: Structures of broken ties: exploring unfollow behavior on Twitter. In: Proc. of the 2013 conference on Computer supported cooperative work, pp. 871-876. ACM, New York, NY, USA, (2013)

53. Hutto, C, Yardi, S, Gilbert, E: A longitudinal study of follow predictors on Twitter. In: Proc. of the SIGCHI Conference on Human Factors in Computing Systems, pp. 821-830. ACM, New York, NY, USA, (2013)

\section{Submit your manuscript to a SpringerOpen ${ }^{\circ}$ journal and benefit from:}

$\checkmark$ Convenient online submission

- Rigorous peer review

- Immediate publication on acceptance

- Open access: articles freely available online

- High visibility within the field

- Retaining the copyright to your article

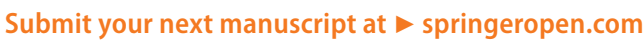

